\title{
O cão como coterapeuta e os cuidados para a sua atuação em ambiente hospitalar
}

\section{The dog as a therapist and care for its performance in the hospital environment}

\section{Thiago de Mello Corrêa}

Graduado em Medicina Veterinária - Universidade do Grande Rio (UNIGRANRIO). Rio de Janeiro, Brasil

Instituição: Hospital Veterinário Cinco Estrelas- Rio de Janeiro, Brasil

Endereço: Avenida Cesário de Melo, 3381. Rio de Janeiro, Brasil

E-mail: thiagomelloesa@gmail.com

\section{Luis Carlos Oliveira Gonçalves}

Mestre em Ciência da Motricidade Humana - Universidade Castelo Branco (UCB), Rio de Janeiro, Brasil; Doutorando em Ciências da Saúde - Universidade Federal de Mato Grosso (UFMT). Faculdade de medicina, Cuiabá, Brasil Instituição: Universidade Federal do Estado do Rio de Janeiro (UNIRIO), Laboratório de Bioquímica de Proteínas

Endereço: Av. Pasteur, 296 - Urca. 22290-240 - Rio de Janeiro, RJ - Brasil

E-mail: luisogoncalves@yahoo.com.br

\section{Aníbal Monteiro de Magalhães Neto}

Doutor em Genética e Bioquímica - Universidade Federal de Uberlândia (UFU) Uberlândia, MG - Brasil

Instituição: UFMT - Instituto de Ciências Biológicas e da Saúde

Endereço: Rodovia MT 100, Km 3,5 s/n. Centro. 78696000 - Pontal do Araguaia, MT - Brasil

E-mail: professoranibal@yahoo.com.br

\section{Adriana da Roza Chaves de Melo}

Doutora em Pesquisa Clínica em Doenças Infecciosas - Instituto de Pesquisa Evandro Chagas (IPEC-FIOCRUZ), Rio de Janeiro, Brasil Instituição: Hospital Veterinário Cinco Estrelas- Rio de Janeiro, Brasil Endereço: Avenida Cesário de Melo, 3381. Rio de Janeiro, Brasil E-mail: adrianachavesvet@gmail.com

\section{RESUMO}

Este estudo teve por objetivo abordar a cinoterapia como modalidade terapêutica capaz de promover uma maior humanização do atendimento ao doente, atuando como ferramenta efetiva no tratamento do assistido, dando ênfase aos cuidados necessários para o emprego do cão no ambiente hospitalar. A humanização da saúde, hoje já bem discutida, apoia iniciativas que visem à transformação do ambiente hospitalar. Acredita-se que, através de pequenas ações, podemos amenizar a dor de muitos, contribuir para o sucesso dos tratamentos e para a diminuição do tempo de hospitalização. A Cinoterapia, apoiada por uma equipe 
multidisciplinar, tem assistido crianças e adultos hospitalizados por diferentes patologias, pacientes cardíacos, psiquiátricos, portadores de Alzheimer, Parkinson, AIDS, paralisia cerebral, acidente vascular cerebral, câncer entre outras. Mesmo diante da tendência mundial e do reconhecimento da importância da terapia, a implantação de projetos ainda tem sido dificultada pela carência de estudos que demonstrem 0 impacto do cão no ambiente hospitalar. 0 desconhecimento dos riscos inerentes à terapia, principalmente no que diz respeito à transmissão de doenças, assim como a falta de protocolos com normas específicas para a sua implantação, são alguns dos entraves para uma maior disseminação da técnica.

Palavras-chave: Cinoterapia, Coterapeuta, Cães.

\section{ABSTRACT}

The aim of this study was to approach Pet therapy as a therapeutic modality capable of promoting a greater humanization of patient care, acting as an effective tool in the treatment of the assisted, emphasizing the necessary care for the use of the dog in the hospital environment. The humanization of health, now well discussed, supports initiatives aimed at transforming the hospital environment. It is believed that, through small actions, we can ease the pain of many, contribute to the success of the treatments and decrease the length of hospitalization. Pet therapy, supported by a multidisciplinary team, has assisted children and adults hospitalized for different pathologies, cardiac patients, psychiatric patients with Alzheimer's, Parkinson's, AIDS, cerebral palsy, stroke, cancer, among others. Even in the face of the worldwide trend and recognition of the importance of therapy, the implementation of projects has still been hampered by the lack of studies that demonstrate the impact of the dog in the hospital environment. The lack of knowledge about the risks inherent to the therapy, especially regarding the transmission of diseases, as well as the lack of protocols with specific norms for its implantation, are some of the obstacles for a greater dissemination of the technique.

Keywords: Pet therapy, Cotherapist, Dogs.

\section{INTRODUÇÃO}

A Terapia Assistida por Animais (TAA) é um recurso terapêutico que se utiliza da relação humano-animal com intuito de promover a saúde física, social, emocional e funções cognitivas das pessoas (DOTTI, 2014). O Brasil está em crescente frente a esse tipo de intervenção, conforme estudo realizado por Santos e Silva (2016), somente no estado de São Paulo foram constatados 29 projetos que utilizam a TAA como terapia complementar, utilizando diversos tipos de animais, além de projetos do Governo Federal - como o Humaniza SUS, 
"cuidando dos cuidadores", "doutores da alegria", mediante os quais crianças passam a aceitar a hospitalização de forma mais positiva, começam a comunicar-se mais, alimentar-se melhor e a colaborar com a equipe de saúde, facilitando assim o trabalho dos profissionais, o que lhes propicia uma melhor qualidade de vida (MAZZETTI, 2005). Atualmente, no Rio de Janeiro, o Projeto Pelo Próximo é o único projeto de TAA que realiza visitas a hospitais públicos do RJ em parceria com a Secretaria Estadual de Saúde.

Mesmo diante da tendência mundial e do reconhecimento da importância da terapia, a implantação de projetos ainda tem sido dificultada pela carência de estudos que demonstrem o impacto do cão no ambiente hospitalar. 0 desconhecimento dos riscos inerentes à terapia, principalmente no que diz respeito à transmissão de doenças, assim como a falta de protocolos com normas específicas para a sua implantação, são alguns dos entraves para a disseminação da técnica. Diante do exposto, esse trabalho objetivou abordar a cinoterapia como modalidade terapêutica capaz de promover uma maior humanização do atendimento ao doente, atuando como ferramenta efetiva no tratamento do assistido, dando ênfase aos cuidados necessários para o emprego do cão no ambiente hospitalar.

\subsection{Interação Homem X Animal}

A utilização dos animais para benefício humano remonta das antigas civilizações a.C.. Os cães ofereciam proteção territorial ao guardar as cavernas contra invasores, além de ajudar nas caçadas. O registro histórico mais antigo dessa relação é a descoberta de um túmulo em Israel datado de 12 mil anos, onde foi encontrado o corpo de uma mulher idosa enterrada com sua mão segurando um filhote de cachorro (DAVIS e VALLA, 1978). Mas é a partir da Revolução Industrial quando os animais começam a não ser tão necessários para as atividades da vida diária, que inicia um processo que durará até os dias atuais, em que se trocou a concepção meramente utilitária para serem vistos como seres amigos (SALAMA, 2005). 
Primeiramente a figura do animal surge nos quintais e com o passar do tempo eles chegam até dentro de casa, como animais de estimação (DOTTI, 2014). Hoje, além de segurança, essa relação adiciona outras necessidades psicológicas (STARLING, et al., 2005). Dentre os fatores que estimularam a necessidade dos animais em casa destacam-se o aumento na expectativa de vida, o aumento do número de pessoas morando sozinhas e o adiamento do plano de ter filhos (SILVA, 2011). Com a convivência os humanos aprenderam a obter vantagens dessa parceria com os animais. De uma mera funcionalidade, 0 homem passou a sentir afeto e percebeu que além de uma função, eles poderiam dar-Ihes algo muito mais valioso, a promoção de saúde (FLÔRES, 2009).

\section{HISTÓRICO}

A citação mais antiga sobre as terapias realizadas por animais data de aproximadamente 400 anos a.C. Hipócrates, grego considerado o pai da medicina, acreditava que cavalgar trazia benefícios neurológicos, e ele estava certo. De acordo com Dotti (2005), há registros no século IX a.C., nos quais Hommer escreveu sobre Asklepios, o deus grego da saúde. Asklepios tinha o poder divino que era estendido a cães sagrados. Acreditava-se que uma pessoa cega poderia voltar a enxergar imediatamente depois de ter sido lambida por um cão sagrado. Na Idade Média havia a crença de que, se uma pessoa pudesse ficar louca, ela deveria então, carregar um cão como amuleto para se prevenir desse acontecimento. Os registros de tratamentos terapêuticos que se utilizavam dos animais aparecem de forma espaçada ao longo do tempo, e inicialmente se davam de forma informal e espontânea, para posteriormente, ganhar organização e reconhecimento.

A utilização de animais como parte de um programa terapêutico foi registrado no século IX, em Gheel, na Bélgica, onde pessoas com necessidades especiais foram autorizadas pela primeira vez a cuidar de animais domésticos. Outros dados, ainda segundo o mesmo autor, apontam que a origem da TAA remonta do século XVIII, na Inglaterra, no retiro de York, uma instituição psiquiátrica que empregava métodos terapêuticos considerados mais humanos para a época. Mantinham nos pátios e jardins frequentados pelos pacientes, 
coelhos, falcões e aves domésticas. Acreditava-se que o contato com eles despertava muito mais que um prazer inocente, mas sentimentos de sociabilidade e benevolência, porém não foram feitos estudos sistemáticos que comprovassem esta hipótese. No século XIX, houve um grande crescimento da participação de animais nas instituições mentais da Inglaterra e demais países europeus e americanos (PEIXOTO, 2009).

Mas, foi a partir da década de 60, que o psicólogo Levinson (1969) iniciou uma série de estudos de situações clínicas nas quais tentava comprovar que a presença de animal era fundamental no processo terapêutico, utilizando o cão como um motivador para crianças que eram resistentes à terapia. Não houve nenhuma outra referência na literatura até que ele descrevesse o uso de animais na prática da Psicologia. Este autor descobriu os efeitos benéficos da terapia com cães, sendo considerado o precursor da Terapia Assistida por Animais (TAA) (DOTTI, 2005).

No Brasil, o primeiro trabalho registrado com animais foi o da psiquiatra Junguiana Nise da Silveira, na década de 1950, que iniciou seu processo de terapia com cães e gatos no hospital psiquiátrico Engenho de Dentro, no Rio de Janeiro, com pacientes esquizofrênicos. Antes mesmo de se pensar em reformar à assistência psiquiátrica, já sustentava a possibilidade do sofrimento psíquico ser passível de expressão simbólica. Para isso, criou ateliês de pintura e modelagem, onde animais - em sua maioria gatos e cachorros - circulavam livremente entre os pacientes (CAPOTE, 2011).

Em 1980 a TAA reaparece no Brasil e o Conselho Federal de Medicina (CFM) reconhece a eficácia do trabalho com cavalos - a Equoterapia - no processo de reabilitação física de uma série de doenças. Mas somente a partir da década de 1990 são fundados os primeiros centros de atendimentos de TAA, marcando o início das pesquisas cientificas sobre o tema (MOTTI, 2007). Por esta ocasião, cães e gatos reassumem o papel de coterapeutas.

Outra precursora em TAA no Brasil é a Dra. Hannelore Fuchs, psicóloga e médica veterinária, que fundou a Associação Brasileira de Zooterapia (Abrazoo) e é coordenadora do "Projeto PetSmile", um serviço comunitário 
filantrópico constituído de visitas de voluntáriose seus animais de estimação, às instituições destinadas às crianças portadoras de deficiências físicas e mentais, idealizado em 1997 (DOTTI, 2005).

De acordo com Santos e Silva (2016) foram criados no ano 2000 os projetos cão idoso e novo guia ambos em São Paulo e com o intuito de admitir a Terapia Assistida por Animais como parte do tratamento dos pacientes e em 2008, foi criado o Instituto Nacional de Ações e Terapia Assistida por Animais (INATAA) e o Centro de Reabilitação e Equoterapia Santo André (CRESA) com a intenção de atender um público diferenciado desde a infância até a velhice.

\section{TERMINOLOGIA}

As intervenções com uso de animais receberam ao longo dos tempos variadas denominações. Levinson (1964) definiu como Pet Therapy (Terapia com Animal de Estimação); posteriormente adotou-se o nome de Pet Psychoterapy (Psicoterapia com Animal de Estimação), delimitando a área de atuação do psicólogo. Surgiram então outros termos como: Human/Companion Animal Therapy, Animal Facilitated Therapy (Terapia Facilitada por Animal) e Zooterapy (zooterapia).

Zooterapia, Atividades Assistida por Animais (AAA), Terapia Assistida por Animais (TAA) e ainda Pet Therapy são denominações para diferentes tipos de assistências humanas onde os animais são utilizados como coterapeutas e coeducadores. Atuam como facilitadores do ensino e aprendizagem e ainda como estimuladores de atividades físicas e terapêuticas (SILVA, 2009).

Atualmente as intervenções com a participação de animais são denominadas: Animal Assisted Activity (Atividade Assistida por Animais - AAA) e Animal Assisted Therapy (Terapia Assistida por Animais- TAA). De acordo com Anderlini e Anderlini (2007), a AAA é uma atividade motivacional, educacional, de lazer, descontração, entretenimento, vínculos, socialização e benefícios emocionais e/ou cognitivos. A atividade não envolve metodologia e/ou procedimentos e o conteúdo da visita é espontâneo. A TAA envolve serviços profissionais da área médica e outras que utilizam o animal como parte do 
trabalho e do tratamento. Segundo Dotti (2005), a TAA é uma intervenção dirigida, específica para cada patologia e faixa etária, onde o animal de estimação especialmente treinado com rígidos critérios de comportamento e saúde é parte integrante do processo de tratamento. Surge como catalisador, modificando o ambiente e o cotidiano do tratamento.

A Delta Society (2005), entidade dos Estados Unidos que regulamenta os programas com o uso de animais, assim definiu: "a Terapia Assistida por Animais (TAA), trata-se de uma intervenção dirigida a um objetivo, na qual o encontro entre o animal e o humano torna- se parte integrante do processo de tratamento; é dirigida por um profissional da área da saúde e está desenhada fundamentalmente para promover melhorias nas áreas física, emocional e social, respeitando o funcionamento cognitivo das pessoas. Pode levar-se a cabo uma ampla variedade de enquadramentos e realizar-se de forma individual ou em grupo. Todo o processo de tratamento deve ter uma avaliação e um registro do mesmo." Os princípios dessa terapia mediada por animais são utilizados em todo o mundo. O trabalho é realizado através da utilização de cães, gatos, cavalos, golfinhos, peixes, tartarugas, coelhos e burros, em hospitais e escolas especializadas no tratamento de pessoas que apresentam problemas psicológicos e na reabilitação de portadores de deficiências múltiplas. Podem ser utilizadas todas as espécies de animais que possam entrar em contato com os humanos sem proporcionar-lhes perigo (KOBAYASHI et al., 2009).

A TAA pode ser aplicada em diferentes faixas etárias e locais, tais como: hospitais, ambulatórios, casas de repouso, escolas, clínicas de fisioterapia e de reabilitação (KOBAYASHI et al.,2009). Esta intervenção deve ser aplicada e supervisionada por profissionais da saúde, devidamente habilitados, sendo todo o processo documentado e avaliado periodicamente (JULIANO et al., 2008).

\section{CINOTERAPIA}

A Cinoterapia é uma modalidade terapêutica que utiliza o cão como instrumento mediador, como facilitador. Quebrando inúmeros paradigmas, os cães vêm demostrando para a comunidade científica que suas aptidões e capacidade de criar vínculo com o ser humano, podem ser grandemente 
aproveitadas nos tratamentos gerando importantes resultados. O termo cinoterapia tem formação da união do prefixo grego "cino" (cão) ao radical terapia (tratamento) (BECKER e MORTON, 2003). A terapia é conduzida por uma equipe multidisciplinar cujos integrantes possíveis incluem médicos veterinários, médicos, terapeutas ocupacionais, fisioterapeutas, enfermeiros, assistentes sociais, fonoaudiólogos, ou profissionais de saúde mental, que se unem para agregar conhecimentos à prática e para planejar ações interventivas adequadas com foco nas potencialidades e não nas dificuldades específicas de cada praticante (BAUN et al., 1991).

Estudos recentes revelam os benefícios incontestáveis do convívio de animais com crianças e adultos, sejam com necessidades especiais ou não, assim como com idosos, presidiários, portadores de enfermidades cardíacas, doentes psiquiátricos, portadores de Alzheimer, Parkinson, Síndrome da Imunodeficiência Adquirida (AIDS), paralisia cerebral, acidente vascular cerebral, câncer, ansiedade, solidão, fobia social, síndrome do pânico e depressão (ANDERLINI e ANDERLINI, 2007). Relatos referentes a algumas patologias demonstram que esta interação cão-paciente melhora o padrão cardiovascular, diminuindo a pressão arterial e os níveis de colesterol. Tal interação também produz o aumento da concentração plasmática de endorfinas, ocitocinas, prolactina, dopamina e diminuem as concentrações plasmáticas de cortisol, substâncias que atuam positivamente no estado de ansiedade (SOBO et al, 2006).

Gonçalves e Gomes (2017) destacam ainda que na interação do homem com o animal, o animal, seja ele de estimação ou não, pode proporcionar ao homem uma série de sentimentos como: fascínio, empatia, sensação de bemestar, amor, afeto e amizade. Com isso, o vínculo estabelecido com a prática da TAA pode auxiliar no combate a depressão, a ansiedade e ao isolamento. A comunicação também é parte do tratamento do paciente e ficar conversando com ele, muitas vezes, é o próprio remédio. Freud já utilizava seu cão durante as consultas e entendia que a simples presença do animal ajudava a tranquilizaro paciente, que conseguia expor melhor seus problemas. O estranhamento dá 
lugar à descontração e o animal abre um canal de comunicação (TELHADO, 2001). O simples fato de tocar uma em um animal pode ajudar a superar a dor de uma pessoa, não apenas fisiológica, mas também a dor emocional (MENCH, 2001).

$\mathrm{O}$ ato de conduzir, escovar, acariciar e jogar bola também ajudam a controlar o estresse, a diminuir a pressão arterial e reduzir os riscos de problemas cardíacos, como comprovado pelo estudo que afirma que a criação dos animais pode causar efeitos relaxantes, evidenciado principalmente pela redução da pressão sanguínea (BAUN et al., 1991). O efeito de acariciar um cão também foi o propósito de um estudo que buscou avaliar em um curto período os resultados da interação de tutores com o animal e os efeitos em acariciar. Os resultados das amostras examinadas constataram a influencia nos níveis de imunoglobulina. Houve um aumento significativo da imunoglobulina $A$ na saliva das pessoas que acariciaram um cão, enquanto que no grupo controle, ao acariciar um cão de pano ou simplesmente permanecerem sentados, não foi observado o mesmo efeito. As atividades realizadas de relaxamento promovem o aumento desse anticorpo, tornando o organismo mais resistente contra invasões de vírus e bactérias (SAVALLI e ADES, 2016).

Lima e Souza (2016) evidenciaram o baixo custo para a aplicação da técnica e o sucesso devido à utilização de animais, já que são amados e admirados por grande parte da população, mas enfatizaram também a importância e a necessidade de maiores pesquisas.

\section{O CÃO COMO COTERAPEUTA}

O cão é o animal mais empregado nas terapias devido à afeição natural pelas pessoas, facilidade de adestramento e por demonstrar mais reações positivas ao toque (PTAK AL, 1995). Além disso, são excelentes companhias, pois durante as visitas não discriminam ou segregam qualquer pessoa, isto é, são livres de preconceitos (MILLER e CONNOR, 2000). Levinson (1962) relata a experiência de um paciente pediátrico com sérios problemas de socialização que abraçado ao cão, discorria sobre suas angústias e aflições. Os resultados 
de seus estudos foram divulgados em um artigo intitulado "The dog as a "cotherapist"' ("O cão como um 'coterapeuta'") (ALTHAUSEN, 2006).

Alguns animais, independente da raça, se destacam por apresentar um temperamento dócil, meigo, paciente, tolerante, equilibrado, tranquilo, firme e estável, características estas, essenciais para garantir o bom andamento do processo terapêutico. Partindo do pressuposto que ambientes como hospitais, escolas, são locais onde é incomum a presença de animais, o emprego de um cão se torna atrativo e motivador.

De acordo com Dotti (2005), a idade ideal para a participação dos cães nos programas de TAA é entre um e nove anos, pois cães muito jovens são geralmente muito agitados. De acordo com Costa et al. (2018), animais muito jovens possuem dentes e unhas afiadas, os muitos velhos se cansam ao término da visita, por isso não devem participar da terapia. Devem apresentar bom comportamento, serem sociáveis com pessoas estranhas e habituadas com o convívio de outros animais (TELHADO, 2001).

Para que o cão possa atuar como coterapeuta, o animal deve ser avaliado por três profissionais, a saber: um veterinário, um psicólogo com especialização em comportamento canino e um adestrador. O primeiro é responsável pela verificação da saúde física do animal; o segundo, pela avaliação do comportamento quanto à socialização, obediência e temperamento do animal; o terceiro vem com a parte do adestramento dos cães, ensinando aos animais como se comportar e usar técnicas e habilidade para lidar com os pacientes que irão participar (DOTTI, 2005). Segundo Anderlini (2009), a socialização do cão como processo de integração de indivíduos é de fundamental importância para essa prática, sendo extremamente necessário que o cão seja socializado para que possa interagir com seres humanos de uma forma coerente e socialmente aceita.

Campos (2009) esclarece que os principais requisitos para um animal terapeuta é demonstrar um comportamento confiável, controlado, previsível, que inspire confiança na pessoa que está interagindo com ele. As contra-indicações se restringem aos animais que demonstrem comportamento de rivalidade e de 
competição com outros animais; animais sem controle de zoonoses podem transmitir doenças e animais mal selecionados que podem causar "acidentes", tais como mordidas, estranhamento e situação de medo (KLEIN, 2007). Os animais testados devem passar por reavaliações constantes (OLIVA, 2004).

É essencial que o animal coterapeuta seja acompanhado em todas as sessões por alguém que se responsabilize exclusivamente por ele, não só por sua atuação nas atividades, mas também pela manutenção de sua segurança e bem-estar durante todo o tempo. Tal condutor deve receber treinamento adequado em comportamento, além de conhecer profundamente a espécie e o indivíduo com quem está trabalhando. É importante que o condutor saiba quais comportamentos podem ser considerados normais para aquele indivíduo eesteja atento para mudanças comportamentais e para exibição de comportamentos que indiquem desconforto (CHELINI e OTTA, 2016). O comportamento canino é tão fascinante para o público quanto para os cientistasque estudam esses animais e cabe ao médico veterinário entender a base fisiológica do comportamento animal (DUKES, 2006).

\section{O CÃO COMO COTERAPEUTA NO AMBIENTE HOSPITALAR}

A prática de envolver animais em hospitais, comum na América do Norte e Europa, ainda engatinha no Brasil. Embora existam registros de casos de sucesso da prática tradicional de visita de cães de voluntários, alguns projetos de lei têm sido apresentados visando consolidar a atividade. O Hospital Albert Einstein em São Paulo é um dos pioneiros da América Latina, tendo passado por testes e treinamentos com equipes de certificação por uma organização americana que reconhece o atendimento de saúde humanizado, para liberação de visita de animais de estimação dos pacientes, mesmo àqueles internados em unidades semi- intensivas (FISCHER et al., 2016).

Segundo critérios do CDC (2003) para que um animal possa ser empregado como coterapeuta em um ambiente hospitalar, será necessária uma avaliação semestral e a apresentação de um atestado de saúde do cão, apresentar comportamento amigável com estranhos e estar habituado com sessões de TAA nesses locais. De acordo com Mcnicholas e Collins (2000), 
durante a presença dos animais nos hospitais, os pacientes têm o nível de ansiedade e estresse reduzidos durante os procedimentos dolorosos, melhoram o relacionamento interpessoal, na promoção do autocuidado, na depressão, no sentimento de solidão, na estimulação da atividade física, nos parâmetros cardiovasculares e no bem-estar. Kawakami e Nakano (2002) observaram também que os pacientes que cuidavam de animais gastavam $16 \%$ a menos de medicamentos e saíam dois dias antes dos hospitais do que os outros pacientes e explica que o contato com animais aumenta as células de defesa e deixa o organismo mais tolerante a bactérias e ácaros, diminuindo a probabilidade de as pessoas desenvolverem alergias e problemas respiratórios. Esses resultados nos permite inferir que cães em hospitais podem reduzir gastos com a saúde pública.

Destacam-se ainda alguns outros benefícios específicos como aperfeiçoar as habilidades motoras finas, o equilíbrio de sustentar-se, a melhora na adesão ao tratamento, o aumento na interação verbal entre os membros do grupo, a melhora nas habilidades de atenção, o desenvolvimento de recreações e lazer, o aumento na autoestima, a redução na ansiedade, na solidão, a melhora na interação com a equipe de saúde e a motivação para o envolvimento em atividades em grupo (FRIEDMAN et al., 1980).

Snipeliski e Burton (2014), em revisão abordando a presença de cães em ambientes hospitalares, os autores destacam os benefícios desse tipo de intervenção, surgindo a partir do aspecto motivacional, auxiliando os pacientes a concentrar-se mais em uma atividade prazerosa e menos nos estressores gerados pela internação. Porque animais de estimação, incluindo cães, geralmente dão aos indivíduos positividade, ajudam a melhorar o humor e reduzir o estresse. Outra questão muito discutida é a percepção da dor. Em uma pesquisa sobre o efeito da TAA nos níveis de dor entre pacientes pediátricos hospitalizados, a percepção dos cuidadores sobre os níveis de dor dos seus filhos mostraram níveis inferiores entre os participantes no grupo intervenção com TAA, quando comparados ao grupo controle, em que os participantes relaxaram em silêncio durante 15 minutos, sem a presença do animal (BRAUN 
et al., 2009). Os pacientes infantis do grupo intervenção relataram sensações de maior bem-estar, causadas pelo aumento na liberação de endorfinas e linfócitos, que, por sua vez, incrementam a resposta imune (BRAUN et al., 2009). Em outro estudo qualitativo, também foi observada uma menor percepção de dor entre crianças hospitalizadas com o uso da TAA (SOBO et al., 2006). Porém, de acordo com entrevistas com os participantes, o consolo, contato físico e sensações do lar que o animal de terapia conseguiu transmitir à criança hospitalizada explicaram esse resultado positivo. Apesar dos estudos citados fornecerem explicações diferentes para a diminuição na percepção de dor da criança, as conclusões apontam para uma menor probabilidade de solicitação analgésicos entre crianças expostas regularmente à TAA.

Savalli e Ades (2016) demostram que a interação com o cão influencia na produção de hormônios nos seres humanos, como a ocitocina e o cortisol. A ocitocina é uma substância produzida pelo hipotálamo para controlar as contrações uterinas no parto e para estimular a produção do leite materno. Os autores destacam que o simples olhar do cão pode provocar um aumento considerável na concentração de ocitocina urinária nos donos de cães. Além disso, existem indícios de efeitos positivos nos comportamentos ligados ao reconhecimento individual e ao apego. Observaram também a diminuição do cortisol.

De acordo com Klinger (2005), a presença de animais no ambiente hospitalar é capaz de interferir no humor das equipes de enfermagem e médica. Além de fornecer benefícios para os pacientes, os cães de terapia podem reduzir o estresse e ansiedade para amigos e familiares que acompanham os pacientes na unidade de saúde. Alguns autores afirmam que a visita dos animais atua melhorando a relação enfermeiro-paciente (LEONOR, 2005). Em estudo recente, Almeida et al. (2016) descreveram a experiência de enfermeiros com o uso desta prática em um hospital oncológico em São Paulo e concluíram que os benefícios vão além do paciente, se estendendo a toda a equipe de saúde. Moreira et al. (2016), abordaram a percepção não somente dos enfermeiros como dos pais, sobre a aplicação da TAA em pacientes pediátricos da oncologia 
e verificaram que ainda há pouca compreensão do funcionamento e dos objetivos da terapia pelos profissionais de enfermagem e familiares, o que dificulta sua implementação de forma rotineira.

\section{RISCOS DA CINOTERAPIA EM HOSPITAIS}

Os animais de companhia são uma fonte potencial para mais de 70 doenças humanas, porém pesquisas com pacientes e estudos epidemiológicos sobre o assunto sugerem que a ocorrência de doença associada a animais domésticos é baixa em geral (STULL et al., 2012).

A presença de animais numa instituição de saúde traz benefícios visíveis a todos os contemplados, entretanto, a companhia animal pode estar associada com a aquisição de doenças no ambiente hospitalar (SILVEIRA et al., 2011). Portanto, torna-se fundamental considerar os riscos e complicações que a transmissão de zoonoses, pode acarretar para os pacientes e para a instituição. As infecções zoonóticas associadas a animais domésticos podem ser adquiridas por meio de mordidas, arranhões ou outros contatos diretos da pele ou membranas mucosas com animais, contato com saliva, urina e outros fluidos corporais ou secreções, ingestão de material fecal animal, inalação de aerossóis ou gotículas infecciosas e através da picada de artrópodes e outros vetores de invertebrados (MANI e MAGUIRE, 2009). Pacientes com comprometimento ou incompleto sistema imunológico desenvolvido, como crianças ( $<5$ anos), idosos ( $\geq 65$ anos), grávidas mulheres e pacientes com condições ou em tratamentos que reduzem a função imunológica estão em risco aumentado de doença associada a animais de estimação (MANI e MAGUIRE, 2009; KOURTIS et al., 2014).

Segundo Pereira et al. (2007), a TAA é contraindicada para pacientes hospitalizados que apresentem alergias, dificuldades de respiração, medo e fobias de animais, pacientes com baixa resistência, com feridas abertas e que apresentem comportamento agressivo, pois podem até machucar e maltratar o animal. Crianças em quimioterapia possuem imunidade comprometida, preocupando, de maneira significativa, os pais em relação ao risco de infecção, gerando apreensão pela presença do cão no hospital. Um estudo sobre o uso da 
TAA em uma unidade pediátrica mostrou que duas crianças não puderam manter um contato prolongado com o animal devido o receio de seus pais quanto ao risco de adquirirem alguma doença (VACCARI e ALMEIDA, 2007). Contudo, outra pesquisa desenvolvida com cães, gatos, coelhos e outros animais em unidade pediátrica comprovou o contrário, mostrando que a imunidade de crianças em quimioterapia tem uma estabilização, principalmente em função dos benefícios trazidos por eles (PAIXÃO et al., 2007).

Uma variedade de patógenos como bactérias (Campylobacter, Salmonella), fungos (dermatophytes), parasitas (Toxoplasma gondii) tem sido identificados como fatores de risco para muitas doenças (CHOMEL, 2014). Sintomas gastrointestinais, principalmente vômitos e diarreias, podem ser observados em infecções causadas por Campylobacter jejuni, que variam sua intensidade de acordo com a condição imunológica de cada paciente, podendo causar até mesmo septicemia. A transmissão se dá através das fezes, estando mais associada a animais jovens. Entretanto, estudos realizados após cinco anos de TAA em ambiente hospitalar, concluíram que o número de infecções não sofreu alteração durante o período que os animais estiveram presentes (KHAN e FARRAG, 2000).

Alguns patógenos são hoje de grande importância na saúde pública, às bactérias multirresistentes, encontradas em humanos e seus animais de estimação (WEESE et al., 2010; LEFEBVRE et al., 2009). Um risco aumentado foi observado em cães que visitam instalações de saúde humana, com a aquisição da Clostridium difficile e Staphylococcus aureus resistente à meticilina, duas a cinco vezes a mais que em cães envolvidos em outras intervenções (LEFEBVRE et al., 2009). De acordo com o CDC (2003), humanos servem como o principal reservatório, mas os animais domésticos são colonizados ou infectados, servindo assim como fonte secundária infecção humana. Estudos sobre infecção hospitalar mostraram ser mais comum um visitante humano transmitir infecções aos pacientes do que os animais, quando devidamente limpos e imunizados. Microrganismos com resistência aos antimicrobianos usuais podem ser transmitidos de pessoas para os animais, promovendo a colonização dos animais por esses agentes (LEFEBVRE et al., 2006). De acordo 
com Friedmann e Son (2009), a lavagem de mãos humana após o contato com um cão de terapia, ajudará na prevenção transmissão da maioria das zoonoses. Em um estudo piloto conduzido por Caprilli e Messeri (2006), não foi observado aumento nas taxas de infecção hospitalar com o início do programade terapia de cães. Da mesma forma, Yamauchi e Pipkin (2008), não encontraram evidências de infecções ou reações adversas em 4.000 pacientesexpostos aos cães de terapia durante um período de seis anos. A recentepublicação de Murthy et al. (2015) inclui um resumo das políticas escritas eprocedimentos de mais de 20 centros de saúde visitados por animais deestimação. Este guia descrevem práticas para minimizar riscos e prevenir a transmissão de doenças zoonóticas.

\section{MEDIDAS PROFILÁTICAS APLICADAS AO COTERAPEUTA}

A saúde do coterapeuta é um aspecto essencial para o bom desempenho e o bem-estar do animal, assim como para a redução do risco de contaminação dos pacientes nos locais de realização das terapias. Gonçalves (2008) orienta que o trabalho deve ser em conjunto com um médico veterinário especialista, pois a cinoterapia alia a parte de saúde pública à fisiologia e comportamento animal. É ele o responsável pela avaliação dos animais sendo o único profissional capacitado para verificar a saúde de um animal terapeuta. Exerce função de orientador, informando e ensinando os cuidados básicos de saúde e higiene de cada espécie, bem como suas particularidades. Ele também deve participar do andamento do projeto para que reavaliações sejam feitas com frequência e o esquema de vacinação e vermifugação respeitados (FLÔRES, 2009).

Segundo critérios do CDC (2003), os cães devem ser avaliados periodicamente, a cada seis meses e apresentar certificado de saúde. Deve também ser vacinado e receber tratamento antiparasitário intestinal periodicamente, não podendo ser portador de Salmonella spp, Campylobacter spp ou Giardia spp, ou até que estejam tratados e tenham teste negativo para as mesmas. De acordo com Flôres (2009), dentre as sugestões de manejo 
sanitário estão a vacinação anual com vacina polivalente, antirrábica, Traqueobronquite Canina, vermifugação a cada quatro meses com medicamentos de amplo espectro, exame parasitológico semestral sempre com resultado negativo, controle de ectoparasitas.

Como rotina de higienização prévia dos animais, além dos banhos é recomendada a escovação, a limpeza de conduto auditivo e escovação dentária, antes de cada visita terapêutica (OLIVA, 2004). Segundo critérios do CDC (2003), além do banho previamente às visitas, com menos de 24 horas, são recomendas também tosas periódicas, conforme o tipo e a raça do animal. Alterações de caráter essencialmente estético tais como pequenas lesões de pele, secreções oculares e até mesmo áreas de tricotomias são fatores limitantes, pois podem causar repulsa ou resistência por parte dos pacientes ou de seus responsáveis em se aproximar dos cães ou qualquer sintoma de doença que possa vir a trazer risco para o paciente assistido e/ou mal-estar para o animal deve ser motivo para o afastamento temporário ou definitivo deste, de suas atividades junto ao grupo (OLIVA, 2004).

De acordo com Silva (2011) para prevenção de riscos é mandatário que a Comissão de Controle de Infecção Hospitalar $(\mathrm{CCIH})$ implemente e monitore estratégias que minimizem essas exposições, isto é, com a adoção de medidas e critérios de segurança para todos os envolvidos (SILVEIRA et al., 2011).

\section{DIRETRIZES PARA A IMPLANTAÇÃO DOS PROGRAMA}

No Brasil os cães empregados nas TAA são considerados cães de companhia e não têm o direito de permanecer em quaisquer locais públicos ou privados. Em relação à legislação ainda não há qualquer regulamento oficial. Atualmente existe apenas um projeto de lei, PL 4455/2012, requisitando o uso de TAA em hospitais públicos cadastrados no Sistema Único de Saúde, porém sem definir ou especificar os requisitos e cuidados necessários para estetrabalho (Rocha, 2015). Para tornar viável que a cinoterapia aconteça dentro das Instituições de saúde, é necessária a elaboração de protocolos abordando as medidas profiláticas a serem adotadas aos cães e a toda equipe envolvida no projeto. 
De acordo com Glenk et al. (2014) devido à falta de consenso em relação aos requisitos de saúde, e especialmente em relação ao comportamento do animal, é indispensável para uma pesquisa científica a descrição precisa do processo de avaliação de saúde, avaliação e seleção dos indivíduos animais (GLENK et al., 2014). Lefebvre et al. (2008) introduziram diretrizes para o emprego de animais nos Programas de cinoterapia nas Instalações de saúde. Entre as diretrizes incluídas destacam-se as recomendações para o treinamento adequado dos manipuladores dos animais e a gestão adequada durante todo o seu envolvimento nesses programas. Em estudo recente, Pam-Hardin et al. (2016), propuseram estratégias visando minimizar o risco de infecções transmitidas em programas de terapia animal, baseadas em 16 anos de experiência. Foram elaborados protocolos com diretrizes para atuação de toda a equipe envolvida na atividade, além dos animais. Durante esse período, nenhuma taxa de transmissão de infecção foi documentada. Segundo o CDC (2003), para a implantação da atividade em uma instituição de saúde, é recomendável a concordância prévia do corpo clínico responsável pela unidade hospitalar onde se pretende implantar a TAA e limitar o acesso dos animais nas áreas de preparação de alimentos e medicação, lavanderia, central de esterilização e desinfecção, sala de cirurgia e de isolamento.

Palley et al. (2011) evidenciam que não há um protocolo padrão para operar um programa de terapia de cães em um hospital e nem o conhecimento real dos riscos da introdução dessa atividade, o que coibe a prática em muitos dos estabelecimentos do país. A maioria dos hospitais em todo o mundo limita a visita animal apenas ao companheiro canino, devido aos menores riscos de infecção e lesão quando comparados a outras espécies.

\section{BEM-ESTAR ANIMAL}

O bem-estar animal é a condição fisiológica e psicológica na qual o animal de companhia é capaz de adaptar-se comodamente ao entorno, podendo satisfazer suas necessidades básicas e desenvolver suas capacidades conforme a sua natureza biológica (CALDERÓN, 2010). O tema tem sido bastante pesquisado ao redor do mundo e, como consequência, produzido conhecimento 
capaz de fornecer subsídio para a elaboração de diretrizes para os diversos tipos de utilização de animais na terapia assistida (COSTA et al., 2018).

No Brasil legalmente os animais são protegidos pela Lei de Crimes Ambientais (Brasil, 1998) que penaliza todo ato de crueldade e maus-tratos contra qualquer animal, além de considerar ilegal a captura e manutenção de animais selvagens sem a autorização dos órgãos competentes. A utilização de animais em atividades acadêmicas é normatizada pela Lei Arouca (Brasil, 2008) que considera ilegal toda atividade com animais que tenha alternativas ou que haja promoção de sofrimento, sem justificativa e aval de um comitê de ética consolidado. Ressalta-se a importância de estudos mais aprofundados e o fato que a ANVISA ainda não possui recomendações sobre a presença de animais em instituições de saúde. A aplicação do princípio ético da precaução embasa a exclusão das terapias de fêmeas no cio, grávidas e com filhotes, assim como animais antissociais, agressivos e com sinais de infeções (SILVEIRA et al., 2011).

A base moral da zooterapia visa garantir o direito dos animais levando em consideração se são usados ou explorados, ressaltando que é repudiado injuriálos na

manipulação; não promover seu bem-estar básico; e forçá-lo a gostar da visita (ZAMIR, 2006). De acordo com Grandin e Johnson (2009), o bem estar do animal está conectado ao bem estar do humano. É natural, então, que se o assistido sente prazer durante a interação com o animal, este, por sua vez, também deverá apreciar a atividade.

Palley et al. (2011) defendem a existência de comitês de cuidado com animais em todos os locais de AAA, que revisassem protocolos e determinassem limites quanto à duração e frequência dos trabalhos tendo em vista o controle de impactos deletérios para os animais. Segundo Odendaal (2000), a terapia também reverte em benefícios para o cão atestado pelo aumento de endorfina, ocitocina, prolactina, ácido fenilacético e diminuição do cortisol e pressão sanguínea. Porém, a indicação de correlação entre o ato de lamber o lábio e o aumento de cortisol, pode fornecer insights sobre o bem-estar de cães em TAA 
e contribuir para aumentar os padrões de educação, certificação e da qualidade de vida desses animais.

\section{CONCLUSÕES}

A Cinoterapia, mesmo consagrada em diversas partes do mundo, ainda encontra grande resistência em muitos dos hospitais do país, principalmente por não permitirem a entrada de animais. Entretanto, é consenso no meio acadêmico que a terapia constitui uma relevante ferramenta para a promoção da humanização do ambiente hospitalar, proporcionando benefícios a toda equipe médica, familiares, pacientes e ao próprio cão, que atua de forma exemplar como coterapeuta, influenciando sobremaneira na abreviação do tempo de hospitalização e dos traumas gerados por ele. Indiscutivelmente, frente à imunossupressão, procedimentos cirúrgicos, bactérias multirresistentes, tornase fundamental que a entrada dos animais em uma unidade hospitalar esteja embasada em normas e critérios de segurança para que os riscos sejam minimizados e os objetivo alcançados. 


\section{REFERÊNCIAS}

ALMEIDA, F.A., NASCIMENTO, A.A. DUARTE, A. M. Terapia Assistida por Animais: A Experiência dos Enfermeiros com o Uso Desta Prática em um Hospital Oncológico. Investigação Qualitativa em Saúde//Investigación Cualitativa en Salud//Volume 2. Atas CIAIQ2016.

ALTHAUSEN, S. Adolescentes com síndrome de Down e cães: compreensão e possibilidades de intervenção. 2006. 170 f. Dissertação de mestrado - Instituto de Psicologia, Universidade de São Paulo, São Paulo.

ANDERLINE, G.P.O.S. Cão-guia, muito mais do que uma companhia: Uma profissão.

Revista do Conselho Federal de Medicina Veterinária, v.15, n. 47, 2009.

ANDERLINE, G.A.O.S.; ANDERLINE G.A. Benefícios do envolvimento do animal de companhia (cão e gato) na terapia socialização e bem estar das pessoas e 0 papel do Médico Veterinário, Revista do Conselho Federal de Medicina Veterinária, n.41, p.70-75, 2007.

BAUN, M.M; OETTING, K; BERGSTROM, N. Health benefits of companion animals in relation to the physiologic indices of relaxation. Holistic Nursing Practice, v.5, n.2, p. 16-23, 1991.

BRASIL (1988). Constituição da República Federativa do Brasil de 1988.

BRASIL Lei complementar ํㅜ 11.794, de 8 de outubro de 2008. Procedimentos para o uso científico de animais.

BRAUN C, STANGLER T, NARVESON J, PETTINGELL S. Animal-assisted therapy as a pain relief intervention for children. Complementary Therapies in Clinical Practice, v. 15, p. 105-109, 2009.

BECKER, M. MORTON, D. O poder curativo dos bichos: como aproveitar a incrível capacidade dos bichos de manter as pessoas felizes e saudáveis. RJ: Bertrand Brasil, 2003. CALDERÓN, N. Bienestar Animal. Revista de la Academia Colombiana de Ciencias Veterinarias, v.1, p.50, 2010.

CAMPOS, P.R.C. O tratamento e ajuda através dos animais. Disponível em:<http://www.slideshare.net/hospvetporto/o-tratamento-e-ajuda- atravs-dosanimais2009>.Acesso em: 22 agosto 2011.

CAPOTE, P.S.O.; Terapia Assistida por Animais (TAA): aplicação no desenvolvimento psicomotor da criança com deficiência intelectual. São Carlos: EdUFScar. 2011. 
CAPRILLI, S., \& MESSERI, A. Animal-assisted activity at A. Meyer Children's Hospital: A pilot study. Evidence-Based Complementary and Alternative Medicine, v.3, n.3, p. 379- 383, 2006.

CHELINI, M.O.M \& OTTA, E. Terapia assistida por animais. Barueri:Manole, 2016. CHOMEL, B. Emerging and re-emerging zoonoses of dogs and cats. Animals, v.4, p. 434-45, 2014.

COSTA, M. P., GATO, F., Rodrigues, M.N. Utilização de terapia assistida por animais como ferramenta no tratamento de doenças em humanos: Revisão PUBVET, v.12, n.1, p.1-7, 2018.

DELTA SOCIETY. Atividade e terapia assistida por animais. A/TAA 2005. Disponível em: <http:// www.projetocao.com.br/main.htm>. Acesso em: 05 agosto de 2011.

DAVIS, S.J.M.; VALLA, F. R. Evidence for domestication of the dog 12,000 years ago in the Natufian of Israel. Nature, v.276, p. 608-610, 1978.

DOTTI, J. Terapia e Animais. São Paulo: Livrus, 2014.

DOTTI, J. Terapia e animais: atividade e terapia assistida por animais - A/TAA: práticas para organizações, profissionais e voluntários. São Paulo: Noética, 2005.

DUKES, H.H. Fisiologia dos Animais Domésticos. 12 Ed. Rio de Janeiro: Guanabara Koogan, 2006.

FISCHER, M. L., AMORIM ZANATTA, A. y REZENDE ADAMI, E. Um olhar da bioética

para a zooterapia. Revista Latinoamericana de Bioética, v.16, n.1, p. 174-197, 2016.

FLÔRES, L.N. Os benefícios da interação homem animal e o papel domedico veterinário. 2009. 34 f. Trabalho de conclusão de curso (Graduação) Universidade Federal Rural do Semi-Arido, Mossoró.

FRIEDMANN, E., \& SON, H. (2009). The human-companion animal bond: how humans benefit. Veterinary Clinics of North America Small Animal Practice 39, 293-326. doi: 10.1016/j.cvsm.2008.10.015.

FRIEDMAN, E. et al; Animal Companions and one-year survival of patients after discharge from a coronary care unit. Public Health Reports, v.95, p. 307-312, 1980.

GLENK, L. M., KOTHGASSNER, O. D., STETINA, B. U., PALME, R., KEPPLINGER, B., 
\& BARAN, H. Salivary cortisol and behavior in therapy dogs during animalassisted interventions: A pilot study. Journal of Veterinary Behavior: Clinical Applications and Research, 9(3), 98-106, 2014.

GONÇALVES, H.J. Seleção e cuidados com os animais terapeutas. Disponível em:. Acesso em: 10 agosto 2008.

GONÇALVES, J.O., GOMES, F.G.C. Animais que curam: a terapia assistida por animais.

Revista UNINGÁ Review, v.29, n.1, p. 204-210, 2017.

GRANDIN, T., \& JOHNSON, C. (2009). Animals in translation: Using the mysteries of autism to decode animal behavior (A Harvest Book). [S. I.]: Simon and Schuster.

Guidelines for environmental Infection Control in Healthcare Facilities. Recommendations of CDC and the Healthcare Infection Control Pratics Advisory Committee (HICPAC). U.S. Department of Health and Human Service Centers for Disease Control and Prevention (CDC). Atlanta: Centers for Disease Control; 2003.

JULIANO, R.S, FIORAVANTI, M.C.S, PAULO, N.M, ATHAYDE, I.B. Terapia Assistida por Animais (TAA): uma prática multidisciplinar para o benefício da saúde humana. [citado 15 jun 2008]. Disponível em: URL: www.vet.ufg.br/.

KHAN, M.A, FARRAG, N. Animal-Assisted Activity and Infection Control implications in a Healthcare Setting. London: St Georges Hospital; 2000.

KAWAKAMI, C.H. \& NAKANO, C.K. Relato de experiência: terapia assistida por animais (TAA) - mais um recurso na comunicação entre paciente e enfermeiro. Simpósio Brasileiro Comum de Enfermagem, 2002.

KLEIN, M. Z. Possíveis benefícios da relação criança/equino na Equoterapia. 2007. 56 f. Trabalho de conclusão de curso (Graduação) - Faculdade de psicologia, Universidade do Vale do Itajaí, Biguaçu.

KLINGER, K. Pesquisas mostram benefícios do convívio com animais. Jornal Folha de São Paulo, 2004.

KOBAYASHI, C. et al. Desenvolvimento e implantação de Terapia Assistida por Animais em hospital universitário. Revista Brasileira de Enfermagem, v.62, n.4, p. 632-636, 2009.

KOURTIS. A.P., READ, J.S., JAMIESON, D.J. Pregnancy and infection. The New England Journal of Medicine, v. 370, p. 2211-2218, 2014.

LEFEBVRE, S.L. et al. Incidence of acquisition of methicillin-resistant Staphylococcus aureus, Clostridium difficile, and other health-care-associated 
pathogens by dogs that participate in animal-assisted interventions. Journal of American Veterináry Medical Association, v.234, p. 1404-1417, 2009.

LEFEBVRE, S.L. et al. Guidelines for animal-assisted interventions in health care facilities. American Journal of Infection Control, v.36, n.2, p. 78-85, 2008.

LEFEBVRE, S.L. et al. Characteristics of programs involving canine visitation of hospitalized people in Ontario. Infection Control \& Hospital Epidemiology, v. 27, n.7, p. 754-758, 2006.

LEONOR, J.M. Visita terapéutica de mascotas en hospitals. Revista Chilena de Infectologia, v.22, n.3, p. 257-63, 2005.

LEVINSON, B.M. The dog as a co-therapist. Mental Hygiene, v.46, 1962.

LEVINSON, B.M. Pet-oriented child psychotherapy. Springfield, IL: Charles C. Thomas, 1969.

LEVINSON, B.M. Pets: a special thecnique in child psychoterapy. Mental Hygiene, v. 48, p. 243-248, 1964.

MANI, I., MAGUIRE, J..H. Small animal zoonoses and immuncompromised pet owners. Topics in Companion Animal Medicine, v. 24, p. 164-74, 2009.

MAZZETTI, M. (2005). Especialistas garantem benefícios de humanização de hospitais. Disponível em:. (Acesso em 22/12/05).

MCNICHOLAS, J., COLLINS, G.M. Dogs as catalyst for social interactions: robustness of the effect. British Journal of Psychology, v., n.1, p. 61-70, 2000.

MENCH, C. Lottie. Disponível em: <http://www.therapydogs.com>. Acesso em: 05 nov. 2001.

MILLER J, CONNOR K. Going to the dogs...for help. Nursing, v.30, n.1, 65-67, 2000.

MOREIRA, R.L. et al. Assisted therapy with dogs in pediatric oncology: relatives' and nurses' perceptions. Revista Brasileira de Enfermagem, v.69, n.6, p. 11221128, 2016.

MOTTI, G.S. A prática da Equoterapia como tratamento para pessoas com ansiedade. 2007. 115 f. Dissertação (Mestrado)- Faculdade de psicologia, Universidade Católica Dom Bosco, Campo Grande.

MURTHY, R. et al. Animals in healthcare facilities: recommendations to minimize potential risks. Infection Control \& Hospital Epidemiology, v.36, n.5, p. 495-516, 2015. 
ODENDAAL, J.S.J.. Animal-assisted therapy-magic or medicine? Journal of Psychosomatic Research, v.49, n.4, p. 275-280, 2000.

OLIVA, V.N.L.S. A terapia assistida por animais: o papel do médico veterinário. Boletim Informativo [documento na Internet] 2004; 34 [citado 2005 Fev 20]. Disponível em: www.anclivepa sp.org.br/rev35-01.htm.

PAIXÃO, A.C.T., SILVA, F.M.G., FONSECA, J.R., \& VIANA, V.E. Estratégias de recreação implementadas e mediadas pela enfermagem durante o tratamento de crianças com leucemia. Trabalho de conclusão de curso (Graduação), Faculdade Brasileira Univix, Vitória, 2007.

PAM HARDIN, M.E. et al. Prevention of transmitted infections in a pet therapy program: An exemplar. American Journal of Infection Control, v.44, p. 846-850, 2016.

PEIXOTO, G. C. X. et al. Zooterapia: uma prática essencial. Pubvet, v.3, n.18, p. 79, 2009.

PEREIRA, M. J. et al. Os benefícios da Terapia Assistida por Animais: uma revisão bibliográfica. Saúde Coletiva, São Paulo, v. 4, n. 14, p. 63-66, abr./maio 2007.

PTAK AL. Studies of loneliness: recent research into the effects of companion animals on lonely people [Internet]. 1995 [cited 2009 Nov 20]. Available from: http://www.deltasociety.org/ Document.Doc?id=121.

REED, R.; FERRER, L.; VILLEGAS, N. Curadores naturais: uma revisão da terapia e atividades assistidas por animais como tratamento complementar de doenças crônicas. Revista Latino-Americana de Enfermagem, v.20, n.3, 2012.

ROCHA, C. F. P.G. Avaliação comportamental e endócrina do nível de estresse de cães participantes de intervenções assistidas por animais. 2015. Dissertação - (Mestrado em Psicologia) - Instituto de Psicologia da Universidade de São Paulo. 2015.122 f.

SALAMA, I. T.A.C.A (terapia asistida com animal).

Disponível em: <http://wwwisabelsalama.com/page3.html>. 2005. Acesso em 08 agosto 2011.

SANTOS, A.R.O; SILVA, C.J. Os projetos de terapia assistida por animais no estado de São Paulo. Revista da SBPH, v.19, n1, 2016.

SAVALLI, C., ADES, C. Benefícios que o convívio com um animal de estimação pode promover para saúde e bem-estar do ser humano. In: CHELINI, M. O. M. OTTA, E. Terapia Assistida por Animais. Barueri: Manole, 2016. 
SILVA, J.M. Terapia Assistida por Animais (Revisão de Literatura). 2011. Trabalho de conclusão de curso (Graduação), Universidade Federal de Campina Grande, Patos.

SILVA, R.M. Bem estar animal em programas de zooterapia ou terapia assistida por animais. Pubvet, v.3, n.20, p. 57, 2009.

SILVEIRA, I.R., SANTOS, N.C., LINHARES, D.R. Protocolo do Programa de Assistência Auxiliada por Animais no Hospital Universitário. Revista da Escola de Enfermagem da USP, v. 45, n.1, p. 283-288, 2011.

SNIPELISKY, D., \& BURTON, M.C. Canine-assisted therapy in the inpatient setting. Southern Medical Journal, v.107, n.4, p. 265-273, 2014.

SOBO, E.J., ENG, B., KASSITY-KRICH, N. Canine visitation (pet) therapy pilot data on decreases in child pain perception. Journal of Holist Nursing, v. 24, n.1, p. 51-57, 2006.

STARLING, A., THOMAS, M., GUIDI, M. O significado do animal de estimação na família. 2001. Trabalho de conclusão de curso [texto na Internet]. [citado 2005 Fev10]. Disponível em: http://culturapsi.vila.bol.com.br/animal.htm.

STULL, J.W. et al. Household knowledge, attitudes and practices related to pet contact and associated zoonoses in Ontario, Canada. BMC Public Health, v. 12, p.553, 2012.

TELHADO, J. Animais ajudam a curar doenças. Jornal do Brasil, Rio de Janeiro, 9 set. 2001.

VACCARI, A.M.H., \& ALMEIDA, F.A. A importância da visita de animais de estimação na recuperação de crianças hospitalizadas. Einstein, v.5, n.2, p. 111116, 2007.

WEESE, J.S. et al. Evaluation of Clostridium difficile in dogs and the household environment. Epidemiology and Infection, v. 138, p. 1100-1104, 2010.

YAMAUCHI, T., \& PIPKIN, E. Six years' experience with animal-assisted therapy in a children's hospital: Is there patient risk? American Journal of Infection Control, v.11, n.113, p. 17, 2008.

ZAMIR, T. The moral basis of animal-assisted therapy. Society and Animals, v.14, n.2, p. 179-199, 2006. 